\title{
Lectura y escritura: reflexiones en torno a la inclusión académica ${ }^{1}$
}

Fecha de recepción: 04 de noviembre de 2016 Fecha de aprobación: 24 de noviembre de 2016 Pp. 81-96
Juan Camilo Méndez Rendón ${ }^{2}$

Universidad de Antioquia

camilo.mendez@udea.edu.co

Cómo citar este artículo: Méndez, J. C. (2016). Lectura y escritura: reflexiones en torno a la inclusión académica. Comunicación, cultura y política, 7, pp 81-96.

\section{Resumen}

Lectura y escritura: reflexiones en torno a la inclusión académica es un texto que tiene como propósito mostrar de qué manera el concepto de lectura y escritura académicas pueden abordarse a partir de la idea de inclusión. Las altas tasas de deserción en la educación superior, cifra que ronda el $45 \%$ en la universidad colombiana, generan una preocupación por parte de varios actores que directa o indirectamente inciden en el destino de este nivel de formación. Siguiendo el método de caracterización de una intervención educativa con un grupo focal, se muestra una posibilidad mediante la cual la universidad puede incluir académicamente a los estudiantes más allá de su condición de riesgo. Esto se constituye en un recurso con el cual la universidad puede mitigar el fenómeno del abandono o la deserción universitaria.

\section{Palabras clave}

Educación superior, deserción universitaria, filosofía de la educación, inclusión, lectura y escritura académicas.

\footnotetext{
${ }^{1}$ El presente texto hace parte del proyecto de acción pedagógica Entre pares y subregiones, financiado por la Facultad de Educación de la Universidad de Antioquia, que inició labores en febrero de 2014 y terminó en diciembre de 2015.

2 Magíster en Lingüística de la Universidad de Antioquia. Doctor en Filosofía de la Universidad Pontificia Bolivariana. Docente de la Universidad de Antioquia en de la Facultad de Educación y en la Escuela de Nutrición y Dietética.
} 


\title{
Reading and writing: reflections on academic inclusion
}

\begin{abstract}
Reading and writing: reflections on academic inclusion is a text that aims at showing how the concept of academic reading and writing can be approached from the idea of inclusion. The high desertion rates in higher education, a figure that is around $45 \%$ in Colombian universities, generate concern on the part of several actors that influence, directly or indirectly, the destination of this level of training. Following the method of characterizing an educational intervention with a focus group, a possibility by which the university can academically include the students beyond their risk condition is presented. This is a resource with which the university can mitigate the phenomenon of university dropout or desertion.
\end{abstract}

\section{Keywords}

Higher education, university desertion, philosophy of education, inclusion, academic reading and writing.

\section{Lecture et écriture : réflexions sur le concept d'inclusion académique}

\section{Résumé}

«Lecture et écriture : réflexions autour de l'inclusion académique » est un texte qui montre de quelles manières les concepts de lecture et d'écriture académiques peuvent être abordés à partir de l'idée d'inclusion. Le fort taux de désertion académique dans l'éducation supérieure colombienne, d'environ 45\%, préoccupe les acteurs qui directement ou indirectement influent sur le devenir de la formation supérieure. Utilisant la méthode de caractérisation d'une intervention éducative avec groupe focal, l'article montre qu'il est possible pour l'université d'inclure académiquement les étudiants en condition de risque. Cette méthode constitue un recours permettant à l'université de mitiger le phénomène de désertion universitaire.

\section{Mots clés}

Éducation supérieure, désertion universitaire, philosophie de l'éducation, inclusion, lecture et écriture académique. 


\section{Leitura e escrita: reflexões sobre a inclusão escolar}

\section{Resumo}

Leitura e escrita: reflexões sobre a inclusão escolar é um texto que pretende mostrar como o conceito de leitura e escrita acadêmica pode ser abordado a partir da ideia de inclusão. As altas taxas de evasão no ensino superior, uma figura de cerca de 45\% na universidade colombiana, gera preocupação aos diferentes atores que direta ou indiretamente incidem no destino deste nível de formação. Seguindo o método de caracterização de uma intervenção educativa através de um grupo de foco, apresentase uma possibilidade a qual a universidade pode incluir academicamente aos estudantes apesar da sua condição de risco. Este se constitui em um recurso com o qual a universidade pode atenuar o fenômeno da evasão universitária.

\section{Palavras-chave}

Ensino superior, evasão universitária, filosofia da educação, inclusão, leitura e escrita acadêmica. 


\section{Introducción}

1 jueves 28 de agosto de 2014, dentro del ciclo de foros que celebraba Cla promulgación de los veinte años de la Ley General de Educación, fue presentada en la Universidad de Antioquia la conferencia "Lectura y escritura: reflexiones en torno a la inclusión académica", que se presentó en el segundo de los foros, titulado "Inclusión educativa: diversidad, vulnerabilidades y discapacidades". Esta conferencia, como era de esperarse, estableció una forma de discontinuidad con las otras que fueron presentadas en el mismo evento, ya que el concepto de inclusión, eje temático de esa actividad académica, mantiene para sí lo que podríamos denominar una acepción generalizada, que la refiere casi que exclusivamente a la relación con los conceptos de diversidad, de vulnerabilidad y de discapacidad.

Pues bien, en dicha conferencia, se mostró que el concepto de inclusión puede servirnos también en el ámbito académico, fundamentalmente en la educación superior. De esta manera, la inclusión se piensa como un acto de hospitalidad, de recibimiento, toda vez que el estudiante que llega a la universidad necesita ser vinculado, no desde la forma administrativa que implica el término, sino desde posibilitarle los medios necesarios que le permitan un estar adecuado en el sitio al que llega. Es bien sabido que la relación universidad-sociedadempresa no puede entenderse tomando cada factor por separado; al contrario, en esa relación, cada factor incide directamente en el otro.

Dicho esto, es necesario comprender por qué la universidad, y en general la sociedad, debiese ampliar el espectro conceptual de la inclusión, con el fin de integrar también la inclusión académica, puesto que en ella es posible ver condiciones de diversidad, vulnerabilidad y limitaciones. En lo que sigue del artículo, vamos a restringir nuestras ideas y nuestro análisis al caso de la Universidad de Antioquia. Metodológicamente lo hacemos así, no por un afán de excluir lo que pasa en otras universidades, sino porque esta universidad reúne dos criterios esenciales para desarrollar el ejercicio de investigación: 1) es una universidad pública y 2) la mayoría de su población pertenece a los estratos 1,2 y 3 .

Esos dos criterios nos hacen pensar que en muchas ocasiones la Universidad de Antioquia se constituye en la única posibilidad que tienen 
muchos de los estudiantes que terminan la escuela secundaria y que, debido a la falta de recursos, no pueden acceder a una de las universidades privadas con que cuenta la ciudad. Esta decisión, sin embargo, también está motivada por el reconocimiento que ella tiene por su trabajo en los ámbitos académico e investigativo. Pero, junto con esta gran aceptación, la Universidad de Antioquia, como tantas otras en Colombia, padece un fenómeno socioeducativo al que necesita prestar atención: la deserción universitaria.

El MEN (2012,p. 67) muestra que los porcentajes de deserción universitaria en Colombia oscilan entre 45 y $50 \%$. Esta cifra es reveladora, porque habla del problema que padecen los estudiantes universitarios, pero se suma a unas cifras insuficientes respecto de la cobertura en la educación superior. Estos indicadores muestran que, de cada dos estudiantes que ingresan a la vida universitaria en Colombia, solo uno culmina sus estudios. Vale preguntarse, en consecuencia, ¿qué ocurre con ese otro estudiante que por alguna razón no continuó con sus estudios? Cualquiera que sea la respuesta que pueda dársele a esta pregunta, no podemos desconocer que tal fenómeno tiene consecuencias, no solo académicas, sino sociales, económicas y políticas de unas dimensiones incalculables.

Asimismo, el MEN (2012) analiza cuatro factores que inciden en la deserción estudiantil universitaria: 1) el factor socioeconómico, 2) el factor institucional, 3) el factor personal y 4) el factor académico. De estos, la mayor incidencia en el abandono escolar la tiene el factor académico, y no el económico, como podría pensarse a simple vista. El factor académico se analiza desde el MEN tomando en consideración las variables de tasa de repitencia, área del conocimiento y puntaje en las pruebas censales del Estado. Cabe mencionar, sin embargo, que la relación entre los factores de abandono por cuestiones académicas y económicas es de causalidad, en cuanto:

antes de la implementación del SPADIES, sistema que permitió el seguimiento poblacional a la permanencia de los estudiantes en la educación superior con una medida estándar y a las condiciones que los acompañan, se asociaba los resultados de la deserción estudiantil a un problema de índole netamente económico, relacionado con la imposibilidad del estudiante de garantizar su vinculación al sistema, dadas las dificultades y limitaciones para cubrir sus costos de matrícula 
y sostenimiento. Sin embargo, la evidencia empírica con la cual cuenta hoy el sector, producto de la consolidación de la información de más de dos millones de estudiantes que han ingresado a la educación superior, permite afirmar que las condiciones académicas de entrada pueden llegar a ser más determinantes para pronosticar las probabilidades de deserción de un joven matriculado en un programa de educación superior. (MEN, 2009, p. 86, 87)

Es dentro de tal realidad que vive la universidad colombiana donde nosotros hemos propuesto el concepto de inclusión académica, y la lectura y la escritura como las prácticas que posibilitan ese tipo de inclusión. Este artículo muestra que la lectura y la escritura le permiten al estudiante hacer parte de una comunidad universitaria y académica. Comunidades en las cuales, muy a menudo, se decide gran parte del desarrollo humano y de movilidad social de los individuos.

\section{Metodología}

11 proyecto Entre pares y subregiones es una iniciativa que busca formar

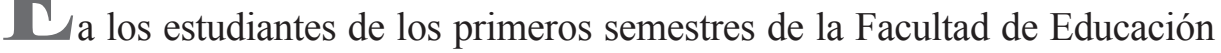
en lectura y escritura académicas, a partir de su participación en seminarios y talleres. Con esto, se busca que el estudiante pueda desarrollar competencias discursivas que le permitan interactuar con los saberes específicos de cada una de las licenciaturas que conforman la facultad. De esa interacción, pueden depender dos cosas en la vida del estudiante: 1) su permanencia y 2) su inserción en una comunidad académica.

El proyecto hace parte del Comité de Permanencia de la Facultad de Educación de la Universidad de Antioquia y es ofrecido y liderado por un grupo de práctica profesional de la Licenciatura en Educación Básica con énfasis en Humanidades y Lengua Castellana. Los estudiantes ${ }^{3}$ inscritos en este grupo

\footnotetext{
3 Dentro de este proyecto, estos practicantes reciben el nombre de pares académicos solidarios, nombre que está en función de la actividad que realizan, pues supone el acompañamiento tutorial con estudiantes de pregrado, especialmente de recién ingreso a la vida universitaria.
} 
de práctica acompañan todo el trabajo de introducción de los estudiantes de primeros semestres (momento en el cual se dan los mayores índices de deserción) a la vida académica, que implica: seminarios y talleres grupales, asesorías general e individual y acompañamiento de proyección académica. Como se ve, aunque la deserción no es el único propósito que abordamos, sí es el que más interés nos genera.

En las jornadas de inducción a los estudiantes admitidos en la cohorte 2014-1, dicho seminario contó con la participación de veinte estudiantes durante dos semanas. Ellos asistieron a un curso intensivo (cuatro horas diarias) que comprendía los siguientes temas:

- Reconocimiento de textos: constituye la apertura en términos generales al proceso tanto de lectura como de escritura en los estudiantes. Desde aquí podemos establecer, a manera de diagnóstico, la relación que existe entre el proceso de comunicar y la tipología empleada, es decir, nos permitió ver el qué en relación con el cómo.

- Lectura crítica: es un eje central en el proceso formativo, pues nos permitió abordar una concepción de escritura y de lectura como dos prácticas que ocurren de manera simultánea. No se dan como procesos dicotómicos. Por otro lado, no nos interesaba una concepción de lectura estrictamente lingüística o cognitiva que sirviera a procesos de desarrollo de un código. De ahí la opción de lo crítico, ya que hay implícita una apuesta por un pensar crítico frente al texto y frente al mundo.

- Argumentación: partiendo de la propuesta esbozada por Nussbaum (2012), su inclusión obedece a dos propósitos: 1) el proceso de pensamiento que se da a partir de la construcción de argumentos razonados y lógicos, y con ello la construcción de ciudadanía que dicha forma de pensar provee; y 2) la posibilidad de debatir los puntos de vista o las posturas frente a los otros, lo cual nos hace pensar en un marco de discusión que cultiva la figura del otro.

- Citación académica (manejo de bases de información): por supuesto que este aspecto lo incluimos por la necesidad que presenta en la escritura académica o de investigación. Con todo, hay una pregunta por lo ético respecto del manejo de la información y la forma como la incorporamos 
en el discurso. De esta manera, el hecho de citar se convierte en una consecuencia natural de un acto ético.

- Escritura científica y de investigación: el criterio de inclusión obedece a que es el tipo de género discursivo que circula mayoritariamente en los ámbitos académicos.

La metodología del curso incluía la escritura de tres textos en tres momentos distintos. El primer texto se escribía en la sesión inicial, y servía como fuente diagnóstica, que nos permitiría ver en ellos el estado de cosas de las categorías preestablecidas. El segundo texto lo escribían en la mitad del curso, y con él se buscaba evidenciar qué elementos nuevos los estudiantes incorporaban a su escritura a partir de lo visto y analizado dentro del seminario. El tercer texto lo harían como el producto final del espacio de formación y buscamos que en este momento se materializara un texto que cumpliera con los criterios textuales académicos desde el punto de vista formal y de contenido.

El tema del texto que debían escribir los estudiantes en aquel momento necesitaba ser estratégico, en la medida en que permitiera que ellos se posicionaran desde un punto de vista particular sobre la temática. El género discursivo era la argumentación, ya que es el que permite la discusión y la confrontación de ideas en el momento de escribir. Tomando en consideración estos dos aspectos, determinamos que los estudiantes escribieran sobre "La explotación sexual". La orientación sobre la escritura del primer texto o texto diagnóstico mencionaba solo que el estudiante pusiese sobre el papel lo que pensaba sobre el tema.

Las categorías de análisis fueron seleccionadas para la valoración de los textos, las cuales coinciden con los ejes teóricos que han inspirado este estudio, de tal manera que el proceso de caracterización de las producciones escritas pudiese ofrecernos información, no solo de las condiciones textuales de la escritura y su forma, sino poder leer en ellas relaciones del sujeto frente al mundo, es decir que no era nuestro interés mirar la escritura desde una perspectiva inmanente, sino como un indicador de conocimiento y ubicación (figura 1). 
Figura 1. Categorías para el análisis textual.

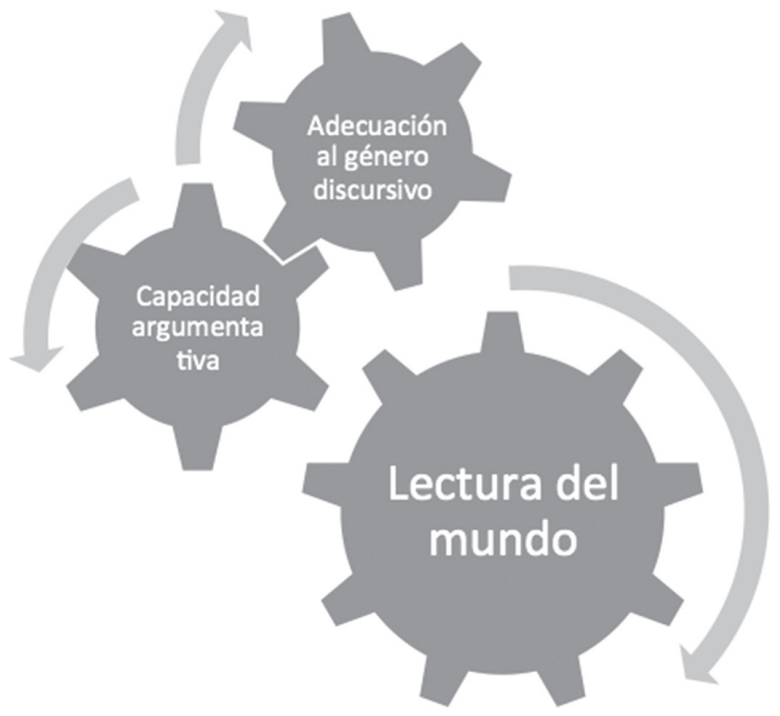

Fuente. Elaboración propia.

De los veinte textos resultantes, hemos seleccionado uno de manera aleatoria para hacer con él el análisis de las categorías. No fue nuestro propósito con esta selección dejar consignada una mirada exhaustiva ni globalizante sobre nuestro objeto de estudio, sino que tan solo pretendimos que, al analizar uno de los textos a través del proceso de escritura, pudiésemos arrojar luz sobre sus consideraciones según las categorías.

También fue nuestro propósito al seleccionar un solo texto darle rostro a un individuo que, de acuerdo con las estadísticas, bien pudiese ser uno de los que en poco tiempo pueda abandonar sus estudios. Esta acción de ponerle nombre propio a la realidad del abandono escolar contrasta con la presentación de los datos estadísticos, donde se difuminan las historias, las realidades y, muchas veces, los infortunios que supone la deserción escolar. 


\section{Componente teórico}

Densar en la inclusión académica nos ha exigido vincular categorías de análisis pertenecientes a esa línea teórica, para explicar desde ellas por qué es conveniente, además de pertinente, volver la mirada a los problemas que ocasiona, entre otros, el abandono escolar y las consecuencias que esto trae en la vida del individuo y de la sociedad en general. De las categorías existentes, como diversidad, discapacidad y vulnerabilidad, nos ha interesado poner el acento en esta última, la cual, dentro de este estudio, sale del cruce de dos variables: la primera la constatación de que hay una relación inversamente proporcional entre estrato socioeconómico y desempeño académico (Isaza, 2011) y la segunda, que se subordina a la primera, es que, por citar tan solo un ejemplo, en la cohorte de ingreso 2015-1 de la Universidad de Antioquia, cerca de $93 \%$ de los admitidos pertenece a los estratos 1, 2 y 3 . Además, de ellos, $73 \%$ proviene de colegios oficiales, mientras que el restante $27 \%$ lo hace de colegios privados (Universidad de Antioquia, 2014).

Cabe hablar, entonces, de exclusiones drásticas, fuertes, finales e inapelables, así como de otras más sutiles, atenuadas o parciales. El término vulnerabilidad o la expresión riesgo de exclusión se utilizan para designar precisamente zonas intermedias entre esos dos polos que, en teoría, cabe establecer entre la exclusión y la inclusión. En ocasiones, ese continuo se personaliza, que es lo que sucede cuando se habla de sujetos en zonas de riesgo o sujetos vulnerables, lo cual supone echar mano de calificaciones que pueden convertirse en etiquetas, cuyos efectos no son, prácticamente nunca, inocentes (Escudero, González y Martínez, 2009, p. 50).

Habiendo esclarecido la razón que nos ha llevado a asimilar la lectura y la escritura como prácticas de inclusión académica, nos proponemos presentar los tres ejes que componen este marco conceptual, considerados en el momento de pensar en el perfil del estudiante que asistiría al seminario y a las características que podía tener al finalizarlo. Nos interesa insistir en que, a pesar de tratarse de un espacio de formación en lectura y escritura académicas, no era nuestro objetivo el aprendizaje mecánico de estas dos prácticas en los ámbitos académicos. Nosotros queríamos, en palabras de Kalman, 
una perspectiva teórica que concibe a la alfabetización (literacy) como algo más que el aprendizaje de los aspectos rudimentarios de la lectura y la escritura; asimismo, se propone que ser alfabetizado refiere a aquella persona que utiliza la lengua escrita para participar en el mundo social. De tal manera, alfabetizarse significa aprender a manejar el lenguaje escrito - los géneros textuales, los discursos, los significados, las palabras, las letras - de manera deliberada e intencional para participar en eventos culturalmente valorados y relacionarse con otros. (2003, p. 39).

Así pues, el primer eje temático que incorporamos proviene de la lingüística, pues debíamos repensar el enfoque que daríamos al seminario de formación en lengua escrita en ámbitos académicos. Ya mencionamos que no perseguíamos el aprendizaje de un nuevo código por el código mismo, ni tampoco queríamos enfocarnos en el nivel gramatical de la escritura. Por consiguiente, nos decantamos, desde el nivel lingüístico, por los aportes de Hymes (1972) respecto de la etnografía de la comunicación. Esta corriente, que comparte territorio conceptual entre la antropología y la lingüística, plantea que la competencia lingüística ha de entenderse como una parte del conjunto de conocimientos y habilidades que componen la competencia comunicativa, a su vez parte de la competencia cultural.

De igual modo, nos inclinamos por los aportes que desde la lectura crítica plantea Cassany (2003). Este autor sostiene que,

ante el mundo multicultural, globalizado, dinámico y conflictivo en el que vivimos, la única respuesta educativa posible es la necesidad de formar a una ciudadanía autónoma y democrática que tenga habilidades críticas de lectura, escritura y pensamiento. (p. 114)

Por otro lado, los otros dos ejes temáticos provienen del campo de la filosofía. Nussbaum $(2005,2012)$, desde la filosofía política, hace énfasis en la argumentación, desde el modelo socrático y el concepto de la vida examinada, no solo como un atributo esencial para la constitución del carácter personal, sino como un aspecto fundamental dentro de una educación humanística, cuyo fin más noble sea preservar los valores fundacionales de la democracia. Este atributo, prosigue Nussbaum (2012), no se alcanza introduciendo diversos 
cursos de filosofía y humanidades en el pénsum de las universidades, sino que solo se alcanza cuando el atributo de la argumentación se convierte en el ethos de las instituciones de educación superior.

Por último, desde la filosofía de la educación, hemos recogido el concepto de lectura del mundo (Freire, 2009). La lectura del mundo freiriana nos permite tomar, en nuestro ejercicio de formación, los conocimientos con que cuentan los estudiantes que llegan a los seminarios y así construir, desde sus necesidades contextuales, la inserción en la cultura académica, a partir de la lectura y la escritura. La lectura del mundo es central en nuestro ejercicio, ya que, como lo sostiene el propio Freire, "es una instancia precedente a la lectura de las palabras. Muchos siglos antes de leer y escribir, los hombres y las mujeres han estado inteligiendo el mundo, captándolo, comprendiéndolo, leyéndolo" (p. 29). De tal forma que la lectura de la palabra en el seminario termina por mejorar la lectura del mundo con que llegan los estudiantes. Aquí reside la razón, por la que, más que un curso de escritura, pensamos en una forma de inclusión académica a partir de la lectura y la escritura en la educación superior.

\section{Discusión}

\subsection{Caracterización sociodemográfica del estudiante}

Código del estudiante $\mathrm{m} 4 \mathrm{f}^{4}$, bachiller de institución educativa del municipio de Guarne, estrato 2, estudiante de la Licenciatura en Matemáticas y Física, primer semestre. La forma en que discutiremos los resultados de la prueba hecha a esta estudiante será como se muestra en la tabla 1. Presentaremos tres fragmentos de cada uno de los textos y en una columna a la derecha haremos el análisis de cada texto desde las categorías consideradas. El análisis lo haremos siguiendo la técnica del comentario.

\footnotetext{
4 El proceso de etiquetación se explica así: la m es la inicial de la licenciatura que cursa (en este caso, matemáticas), el 4 es el orden en que se analizó su producción, y la f corresponde a su género.
} 
Tabla 1. Caracterización y análisis de la producción escrita de un estudiante participante del seminario de formación sobre lectura y escritura académicas.

\begin{tabular}{|c|c|c|}
\hline $\begin{array}{c}\text { Estudiante } \\
\text { m4f }\end{array}$ & Producción escrita & Análisis desde las categorías \\
\hline $\begin{array}{c}\text { Texto } \\
\text { diagnóstico } \\
\text { (1) }\end{array}$ & $\begin{array}{l}\text { "Pienso que la explotación sexual no } \\
\text { le conviene a una persona, porque no } \\
\text { podría llevar una vida normal. Sin } \\
\text { embargo, eso ocurre en nuestro país y } \\
\text { en el mundo, y uno no ve que se haga } \\
\text { algo por solucionar ese tema [...] } \\
\text { hay que participar más en las formas } \\
\text { de prevención de este delito y la } \\
\text { responsabilidad tam-bién la tiene } \\
\text { las autoridades, depende de ellas } \\
\text { que termine, aunque también de la } \\
\text { situación socioeconómica de las } \\
\text { personas". }\end{array}$ & $\begin{array}{l}\text { El diagnóstico evidencia un acercamien- } \\
\text { acercamiento al tema desde lo } \\
\text { general, posiblemente a partir de un } \\
\text { conocimiento, cuyas fuentes provengan } \\
\text { de los medios de comunicación, que no } \\
\text { suelen dar lugar al análisis riguroso ni } \\
\text { particular; no hay posicionamiento del } \\
\text { punto de vista, razón por la cual no se } \\
\text { evidencia argumentación formal, y al } \\
\text { final hay un fragmento que se pierde en } \\
\text { la ininteligibilidad, producto de ideas } \\
\text { confusas ("hay que participar más en } \\
\text { las formas de prevención") y redacción } \\
\text { precaria ("esto que depende de ellas que } \\
\text { termine"). }\end{array}$ \\
\hline $\begin{array}{c}\text { Texto } \\
\text { diagnóstico } \\
\text { (2) }\end{array}$ & $\begin{array}{l}\text { "Este se define como toda actividad o } \\
\text { acto sexual entre dos o más personas } \\
\text { sin consentimiento de una persona; por } \\
\text { otro lado, es una realidad angustiante } \\
\text { con la que muchos tienen que } \\
\text { convivir día a día, es un tema bastante } \\
\text { serio, pero al que le presta poca } \\
\text { importancia, debido a que la sociedad } \\
\text { aún lo ve como un tabú. Es triste ver } \\
\text { que las autoridades, los medios de } \\
\text { comunicación y la sociedad en sí se } \\
\text { nieguen a brindar la ayuda necesaria } \\
\text { y se mantengan tan al margen de estos } \\
\text { casos, y que su único 'aporte' sean } \\
\text { las críticas, sin saber que este tipo de } \\
\text { reacciones agrava el trauma y hace } \\
\text { que la víctima se aísle, dificultándole } \\
\text { el proceso de recuperación". }\end{array}$ & $\begin{array}{l}\text { En este segundo texto, se ve una } \\
\text { apropiación conceptual más decidida. } \\
\text { Se habla no solo desde la perspectiva } \\
\text { propia, sino que se incluyen otras voces } \\
\text { que ayudan a delimitar conceptualmente } \\
\text { el tema. La autora hace uso de lo que } \\
\text { Nussbaum (2005) llama la imaginación } \\
\text { narrativa, es decir, la capacidad de } \\
\text { ponerse en el lugar del otro e imaginar } \\
\text { su sentir. Discute el papel de los medios } \\
\text { frente al tema y critica su acción, al } \\
\text { tiempo que muestra su punto de vista } \\
\text { respecto de la explotación sexual } \\
\text { (argumentación); por último, se aprecia } \\
\text { una escritura muy cuidada, no solo } \\
\text { desde la forma, sino desde el contenido. } \\
\text { En la primera, usa correctamente } \\
\text { la puntuación, la acentuación y la } \\
\text { ortografía; en la segunda, se ayuda } \\
\text { de marcadores discursivos ("por otro } \\
\text { lado", "pero"), lo cual contribuye a que } \\
\text { la idea se presente con mayor fuerza } \\
\text { y claridad. Hay que mencionar que } \\
\text { en este texto y en el tercero los textos } \\
\text { fueron evaluados en la parte formal por } \\
\text { el asesor y los practicantes. }\end{array}$ \\
\hline
\end{tabular}


Tabla 1. Caracterización y análisis de la producción escrita de un estudiante participante del seminario de formación sobre lectura y escritura académicas (Continuación)

\begin{tabular}{|c|c|c|}
\hline $\begin{array}{c}\text { Estudiante } \\
\text { m4f }\end{array}$ & Producción escrita & Análisis desde las categorías \\
\hline $\begin{array}{c}\text { Texto } \\
\text { diagnóstico } \\
\text { (3) }\end{array}$ & $\begin{array}{l}\text { "Hace aproximadamente un año, en } \\
\text { un artículo publicado por Redacción } \\
\text { Cali en el periódico El Tiempo, } \\
\text { una joven madre da su testimonio. } \\
\text { Ella dice lo siguiente: 'La señora } \\
\text { es buena... había cosas que no me } \\
\text { gustaban, pero me enseñó a leer } \\
\text { y le podía mandar plata a mi hijo. } \\
\text { No podía salir a la calle, porque me } \\
\text { decía que era peligroso y cuando ella } \\
\text { se iba me tocaba tener sexo con el } \\
\text { marido' (2013, s. p.). Como ella, hay } \\
\text { miles de mujeres que son explotadas } \\
\text { sexualmente, ¿cómo puede decirse } \\
\text { que es una vida fácil? } \\
\text { Estas mujeres tienen que pasar por una } \\
\text { realidad dura y hostil, sufrir maltratos, } \\
\text { humillaciones, son obligadas a mante- } \\
\text { ner relaciones sexuales, en su mayoría, } \\
\text { sin protección alguna, y en los peores } \\
\text { de los casos, una enfermedad venérea } \\
\text { o hasta la muerte, logrando así brin- } \\
\text { darles ingresos económicos a sus } \\
\text { captores". }\end{array}$ & $\begin{array}{l}\text { Más allá de la importante adecuación } \\
\text { textual al género académico, esta } \\
\text { versión del texto deja ver recursos } \\
\text { retóricos propios del género, como usar } \\
\text { argumentos que incidan en los senti- } \\
\text { mientos personales ("la historia del } \\
\text { periódico"), usar argumentos racionales } \\
\text { ("son víctimas de explotación sexual en } \\
\text { muchas ocasiones porque las secunda } \\
\text { una necesidad económica"). } \\
\text { Se evidencia el recurso de la polifonía } \\
\text { textual que, como ya se dijo, fortalece } \\
\text { la visión propia. La idea sobre la explo- } \\
\text { tación ya no descansa sobre imaginarios } \\
\text { ni estereotipos, sino que trasciende } \\
\text { hacia una realidad causada por algo } \\
\text { y con consecuencias individuales y } \\
\text { sociales serias. }\end{array}$ \\
\hline
\end{tabular}

Fuente. Elaboración propia. 


\section{Conclusiones}

T as universidades son responsables por la formación en lectura y escritura $\bigcup$ de sus estudiantes, lo cual debe acabar con el juego eterno que consiste en que la universidad responsabiliza a la escuela por tal formación, y esta, a su vez, a la familia, y así, en un círculo interminable. Esa responsabilidad implica ofrecer espacios formativos que le permitan al educando formar capacidades con las cuales puede interactuar en comunidades académicas, al tiempo que se forma como ciudadano activo, reflexivo, responsable en su toma de decisiones.

El territorio conceptual de la inclusión educativa, de la cual es sucedánea también la exclusión, requiere expandir sus límites hasta incluir realidades como la inclusión académica y otras de igual necesidad. Solo así podrá captar la atención como un problema que está incidiendo directamente en la organización política, social y económica de Colombia.

La deserción escolar es un problema que el estudiante no ha propiciado. Eso se llama exclusión. El sistema social crea las condiciones para que exista esa exclusión. De ahí que, más allá de esta realidad, la educación está perdiendo su condición de medio para la promoción social. Una leve comparación de resultados en pruebas censales podría hacernos pensar que la educación perpetúa las élites, por eso la necesidad de la inclusión académica. 


\section{Referencias}

Cassany, D. (2003). Aproximaciones a la lectura crítica: teoría, ejemplos y reflexiones. Tarbiya: revista de investigación e innovación educativa del Instituto Universitario de Ciencias de la Educación, 32, 113-32. Recuperado de https://repositori.upf.edu/handle/10230/21224

Escudero Muñoz, J. M., González González, M. T. y Martínez Domínguez, B. (2009). El fracaso escolar como exclusión educativa: comprensión, políticas y prácticas. Revista Iberoamericana de Educación, 50, 41-64.

Freire, P. (2009). El grito manso. México: Siglo XXI.

Hymes, D. (1972). Models of the interaction of language and social life. Nueva York: Basil Blackwell.

Isaza, J. F. (27 julio 2011). Deserción en la educación superior. En El Espectador. Recuperado de http://www.elespectador.com/opinion/ desercion-educacion-superior-columna-287578

Kalman, J. (2003). El acceso a la cultura escrita: la participación social y la apropiación de conocimientos en eventos cotidianos de lectura y escritura. Revista Mexicana de Investigación Educativa, 8(17), 37-66.

MEN (Ministerio de Educación Nacional) (2012). Deserción estudiantil en la educación superior colombiana: metodología de seguimiento, diagnóstico y elementos para su prevención. Bogotá: MEN.

Nussbaum, M. C. (2005). El cultivo de la humanidad: una defensa clásica de la defensa de la educación superior. Barcelona: Paidós.

Nussbaum, M. C. (2012). Sin fines de lucro: por qué la democracia necesita de las humanidades. Buenos aires: Katz.

Universidad de Antioquia (4 diciembre 2014). UdeA recibe a 5,467 estudiantes. Recuperado de https://goo.gl/oMM9pj 